\title{
Improving GSM Services in Nigeria Through Interference Mitigation
}

\author{
${ }^{1}$ Nsionu Ifeanyi, and ${ }^{2}$ Onoh Gregory \\ ${ }^{1}$ Computer Engineering Department, \\ Federal Polytechnic, Oko-Anambra State, Nigeria \\ ${ }^{2}$ Electrical Electronic Engineering Department, \\ Enugu State University of Science \& Technology,Enugu-Nigeria
}

\section{Doi:10.5901/jesr.2013.v3n4p121}

\begin{abstract}
Since the early days of wireless communication till today, there has been the simple dipole (oneelement) antenna which functions by radiating and receiving radio frequency (RF) energy equally well in all directions. This rather unfocussed/indiscriminate approach of radiating RF signals is just adequate for simple RF environments where no specific knowledge of the whereabouts of the gsm user is available. It is very clear that as huge amounts of RF power is radiated into the environment, only a small portion of it is received. Given this limitation therefore, this present and obsolete technique attempts at overcoming its challenges by simply boosting the power levels of the signal which it pushes blindly into the atmosphere. Unfortunately, all the gsm operators in Nigeria and indeed in many countries, have continued to deploy obsolete antenna systems (Omnidirectional and Sector antennas) which are characterized by colossal waste in RF energy and interference. This paper presents the result of an investigation and combination of two techniques of Direction of Arrival (DOA) Estimation and Beam Pattern Synthesis (BPS) for suitable implementation in antenna system, the combination of which will ensure that (a) RF energy is not available in areas where gsm users are non-existent (b) RF energy is available in only the areas where there is at least one gsm user with wireless device turned on. The DOA estimation technique proposed in this paper is the Multiple Signal Classification (MUSIC). For the BPS, the Least Mean Square (LMS) technique is used. Validation of the workings of these two applications, which form the Digital Signal Processing (DSP) algorithm for the antenna system, was carried out using the Matlab as the simulation tool. In the case of the MUSIC technique, the simulation was run for four different signals originating from different directions (or angles) using a fixed number of antenna elements. The results show clearly the ability of the system to identify each gsm signal and its direction of arrival. In the case of LMS technique, simulation was run for two signals, one desired and the other undesired (interferer). The results for the pair of signals are displayed graphically, where the unwanted signal is seen mitigated by the system. The combination of DOA and BPS for implementation in gsm networks ensures that the huge waste in RF energy and interference will be a thing of the past as the energy will be available only in the area needed.
\end{abstract}

Keywords: direction of arrival, beam pattern synthesis, digital signal processing, least mean squares, multiple signal classification

\section{Introduction}

The world allover has continued to witness huge demand for voice, data and video related services. This is despite the current deployed infrastructural facilities in many countries, including Nigeria. Telecommunication service providers in this country have continued to witness unprecedented demand with the result that the infrastructure so far deployed could not provide satisfactory 
services to the people. Therefore these operators continue to face numerous challenges in their quest to make the populace have access to quality telecommunication services.

Two fundamental concerns for gsm operators are (1) how to acquire more capacity so that a large number of subscribers can be served at lower costs (2) how to obtain greater coverage areas so as to reduce infrastructure and maintenance costs. These are issues of spectral efficiency and coverage respectively. At the initial phase of system rollout, coverage (and not capacity) is the important factor, but as the demand overtakes supply, the major issue becomes the network capacity; this gives rise to incessant upgrading activities as witnessed by the subscribers. The term Capacity can be explained from two different sides; from the point of view of the subscriber and the network operator. To the subscriber, capacity is measured by the quality of each successful link he gets and the number of times he can secure such a link whenever he wants it. The subscriber simply wants the best quality link at the lowest possible cost. To the network operator, capacity is the number of successful links that can be simultaneously established. Since the quality and the number of simultaneous links are inversely related in a resource-constrained environment, the operators tend to lean towards providing the lowest possible (mindful of the threshold) quality links to the largest possible number of simultaneous subscribers. So, while the subscriber wants the best links at lower costs, the operators want maximum profits by providing an increasing number of lower quality links at the highest acceptable cost to the subscriber.

The greatest impediment to capacity increase in gsm system is interference and, until the quest for real capacity is achieved (through interference mitigation), the controversy between the subscriber and the operator over capacity will linger on. The crux of the matter is that today's technology in Nigeria can provide for increased range in some cases and up to a limited capacity in other cases, but it rarely can provide both at the same time.

'Space' is one of the final frontiers when it comes to wireless communication. The only way to successfully tackle the issues of capacity, quality and coverage is to abandon the current unfocussed transmission of RF signals using the obsolete antenna systems and embrace the technology of spatially selective transmission and reception of RF energy. This technology entails filtering in space domain which can separate spectrally and temporarily overlapping signals from multiple gsm signals. Thus, spatial dimension can be exploited as a hybrid multiple access technique complementing the already existing ones such as FDMA, TDMA and CDMA. This latest technique is called the Space Division Multiple Access, SDMA. The technology enables multiple users within the same radio cell to be accommodated on the same frequency and time slots.

An antenna system that can employ the SDMA to realize spatial filtering is called Adaptive Antenna. The term 'Adaptive' points to the fact that the antenna system is capable of modifying its frequency, time and spatial response. The deployment of such antenna which exploits the spatial domain ensures the following operational benefits to the network provider:

Enhancement of capacity: the technology allows for multiple users in a cell to use the same frequency without interfering with each other since the antenna beams at the BTS are sliced to keep different users in separate beams at the same frequency

Extension of coverage area: the increase in range is due to the achievement of a bigger antenna gain, the implication of which is the deployment of much fewer BTS to cover a given geographical area.

Increased immunity to interference scenarios.

- $\quad$ The problem of traffic load imbalance would be significantly reduced. Traffic load imbalance is a time-dependent phenomenon where cellular traffic is not evenly distributed among different geographical areas of the network or among the sectors of a site, leading therefore to increased call blocking/call drop. The unused capacity on other sectors/sites is wasted.

\section{Methodology}

Two techniques will be used to realize network capacity increase through interference mitigation 
namely; Direction of Arrival estimation of gsm signal and antenna Beam Pattern Synthesis. Workable algorithms will be developed to achieve these.

\section{A. Direction of Arrival Estimation}

For one given source of a gsm signal, there are many possible propagation paths and angles of arrival. Therefore, for several transmitters operating at the same time, it is apparent that each source creates many multipath components at the receiver. It is important for a receive antenna to be to estimate the directions (or angles) of arrival of the signals the information of which will be used by the antenna to eliminate or combine signals for greater fidelity or suppress interferers or both to improve the capacity of gsm network.

Let us consider several $D$ signals arriving at equally spaced elements of an antenna. Each of the $D$ signals hit the antenna elements with an angle equal to $\theta_{i}$. The goal of the DoA estimation is to use the data received at the array to estimate $\theta_{i}$ where $i=1,2,3, \ldots \ldots, D$.

In this presentation, the Multiple Signal Classification, or MUSIC technique will be used to determine the signal direction of arrival. This method makes the assumption that the noise in each channel is not correlated, making the noise correlation matrix diagonal. The incident signals may be correlated, which creates a non-diagonal correlation matrix for the signal. Given that the number of signals impinging on $M$ antenna elements is $D$, then the number of signal eigenvalues and eigenvectors is $M-D$. The array correlation matrix with uncorrelated noise and equal variances is given by [1]

$$
R_{x x}=A * R_{s s} * A^{H}+\sigma_{n}^{2} I
$$

Where $A=\left[\begin{array}{llll}a\left(\theta_{1}\right) & a\left(\theta_{2}\right) & a\left(\theta_{3}\right) \ldots \ldots & a\left(\theta_{D}\right)\end{array}\right] \quad$ is a $M \times D$ matrix

$R_{s s}=\left[\begin{array}{llll}s_{1}(k) & s_{2}(k) & s_{3}(k) \ldots \ldots . & s_{D}(k)\end{array}\right] \quad$ is a $D \times D$ source correlation matrix

$R_{x x}$ has $D$ eigenvectors associated with signals and $M-D$ eigenvectors associated with noise.

We can construct the $M \times(M-D)$ subspace spanned by the noise vectors such that

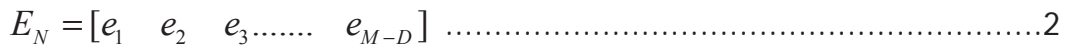

The noise space eigenvectors are orthogonal to the array steering vectors at the angles of arrival $\theta_{1}, \theta_{2}, \theta_{3}, \ldots \ldots . . \theta_{D}$. From the point of view of the orthogonality condition, it can be shown that the Euclidean distance

$$
d^{2}=a(\theta)^{H} E_{N} E_{N}^{H} a(\theta)=0
$$

For each and every arrival angle $\theta_{1}, \theta_{2}, \theta_{3}, \ldots \ldots . \theta_{D}$. Placing this distance expression in the denominator creates sharp peaks at the angles of arrival indicative of the required DoA. The MUSIC formula is now given as

$$
P_{(\text {MUSIC) }} \theta=\frac{1}{a b s\left(a(\theta)^{H} * E_{N} * E_{N}^{H} * a(\theta)\right)}
$$

Next, we develop a sample calling program designed to validate the working of the MUSIC algorithm. The sample program is simulating the input that is arriving at the array. The array itself is an $m$ (NOOFELEMENTS) element linear array with no directional properties in the given plane. The number of elements can be varied. Further, the frequency of the input can be changed. Dfactor is the division factor of the wavelength which represents the distance between the antenna elements. For example, Dfactor $=2$ is the distance between elements $=\lambda / 2$. Thus, we have a handle on both the frequency and the distance between elements in terms of the given frequency. The samples variable represents the number of samples that we wish to take for the given input and $\mathrm{f}_{\mathrm{s}}$ is the sampling frequency. These two variables can be adjusted to stimulate the processor 
available, the level of accuracy and system refresh rate. Choosing a high value of samples would lead to better accuracy of calculations.

The matrix below of $x$ is the form in which the signal received at the antenna elements is stored.

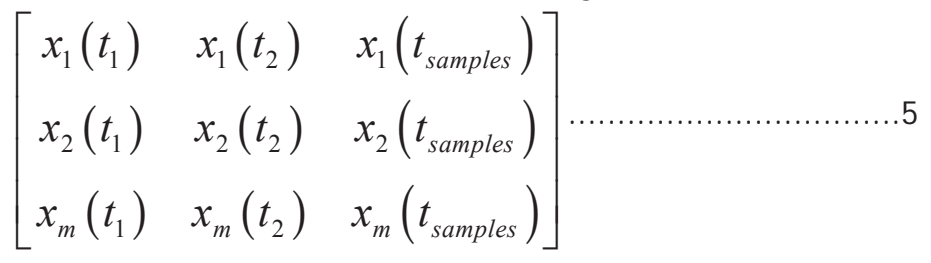

where $x_{i}\left(t_{j}\right)$ represents the value of the input function detected at the $i_{t h}$ element at the $j_{t h}$ time instant, (including noise). Once the array manifold function is defined in a different file, all that is needed is to call the $m u s i c 1 d$ function to obtain the estimate of the number of signals and plot the $P_{M U}(\theta)$ versus $\theta$.

The array manifold is defined as the gain and phase change provided by the array elements to a given signal direction. The array manifold is a function of $\theta$ and is saved in the manifold file. The variables NOOFELEMENTS and dfactor are global and hence can be assessed by the manifold too.

The concluding part of the MUSIC algorithm consists of the actual program implementation. The function takes as input the $x$ matrix from which the number of elements and samples can be seen. The first step is the calculation of the eigenvalues and the eigenvectors of the matrix. The next step is to obtain the noise eigenvector matrix of the minimum eigenvalues and form the matrix $n e v$. Using this $n e v$, we can plot to obtain the direction of arrival in a single or multiple directions. The final aim is to get DOA in terms of the angles of azimuth and elevation.

\section{B. Beam Pattern Systhesis}

One criterion which can be applied to enhance the quality of the received signal and at the same time reduce the influence of the interfering signal is based upon the maximization of signal-tointerference ratio (SIR). If we can cancel all interfering signals by placing nulls at their various directions of arrival, then we would have maximized the SIR. Blocking of interference signals (nulling) and acceptance of the desired signals (enhancement) is achieved by systematic assignment of weight calculated for each antenna element by the system. This activity is done in the DSP unit of the antenna. The weights calculated for each element can either be fixed (fixed weight beamforming) or adaptive (adaptive beamforming). Before demonstrating the latter, it is necessary to show how fixed weight beamforming can be achieved for non-adaptive antenna.

\section{Interference Control By Fixed Weight Beam Forming}

Let us consider an antenna system comprising three elements meant to receive a fixed gsm signal accompanied by undesired interferers as follows: $\theta_{o}=$ angle of arrival for the desired signal from a fixed source, $\theta_{1}=$ angle of arrival for the first accompanying interfering signal, $\theta_{2}=$ angle of arrival for the second accompanying interfering signal. $\theta_{0}, \theta_{1}, \theta_{2}$ are all operating at the same carrier frequency. The array vector is given by [1]

$$
a=\left[\begin{array}{lll}
e^{-j k d \sin \theta} & 1 & e^{j k d \sin \theta}
\end{array}\right]^{T}
$$

For optimization, the array weights are given by

$$
w^{H}=\left[\begin{array}{lll}
w_{1} & w_{2} & w_{3}
\end{array}\right]
$$


So that the total array output is

$y=w^{H} \cdot a=w_{1} e^{-j k d \sin \theta}+w_{2}+w_{3} e^{j k d \sin \theta}$

Three array outputs are involved here.

$y_{o}$ is the array output for the desired signal

$y_{1}$ and $y_{2}$ are the array outputs for the interfering signals. It is natural that three conditions must follow the three unknown weights $w_{1}, w_{2}, w_{3}$ as follows

$$
\begin{aligned}
& y_{o}=w^{H} \cdot a_{o}=w_{1} e^{-j k d \sin \theta_{o}}+w_{2}+w_{3} e^{j k d \sin \theta_{o}}=1 \\
& y_{1}=w^{H} \cdot a_{1}=w_{1} e^{-j k d \sin \theta_{1}}+w_{2}+w_{3} e^{j k d \sin \theta_{1}}=0 \\
& y_{2}=w^{H} \cdot a_{2}=w_{1} e^{-j k d \sin \theta_{2}}+w_{2}+w_{3} e^{j k d \sin \theta_{2}}=0
\end{aligned}
$$

The first condition which requires $y_{o}$ to be 1 means that the desired signal will be received in full without any modifications. The other two conditions signify the rejection of the interfering signals. These conditions can be stated in matrix form as follows:

$$
w^{H} \cdot A=u_{1}^{T}
$$

To find the weights, we find the inverse of $A$ and then multiply by the Cartesian basis vector transpose;

$w^{H}=u_{1}^{T} \cdot A^{-1}$

This last equation enables the calculation of the array weights for beamsteering. The number of the array weights depends of course on the number of elements. For $M=16$ elements, 16 array weights $w_{1}, w_{2}, \ldots \ldots, w_{16}$ shall be calculated. Both the desired and interfering signals are received by the array of $M$ elements with $M$ potential weights.

\section{Interference Control By Adaptive Beamforming}

The fixed weight beamforming earlier discussed shows that as long as the signal direction of arrival does not change with time, then the calculated optimum weights will not need to be adjusted. However, if the desired angles of arrival change with time, (as in the case of a mobile terminal), it becomes very necessary to devise an optimization scheme that is capable of re-calculating the optimum array weights. The implication of this is that the received signal processing algorithm must allow for the continuous adaptation to ever-changing signals. Now, the adaptation process must satisfy a specific optimization criterion. In this paper, the least mean squares criterion will be used.

\section{The Least Mean Squares (Lms) Criterion}

An alternative means for array weights optimization is by minimizing the mean-square error, MSE. This involves minimizing the error while iterating the array weights. Given a reference signal $d(t)$ which is either identical to the desired signal $s(t)$ or is highly correlated with the desired signal while at the same time uncorrelated with the interfering signal $i_{n}(t)$. If $s(t)$ is not distinctively different from the interfering signals, the minimum mean square error method will not work properly. Now, the error signal $\varepsilon(t)$ is such that

$$
\varepsilon(t)=d(t)-w^{H}(t) x(t)
$$

The squared error is given as

$$
|\varepsilon(t)|^{2}=\left|d(t)-w^{H}(t) x(t)\right|^{2}
$$


By some algebraic analysis, it can be shown that the MSE is given by

$$
|\varepsilon(t)|^{2}=|d(t)|^{2}-2 d(t) w^{H} x(t)+w^{H} x(t) x^{H}(t) w
$$

Leaving behind the time dependence notation $(\mathrm{t})$, we take the expected value of both sides and simplify to obtain the expression

$$
\begin{aligned}
& E\left[|\varepsilon|^{2}\right]=E\left[|d|^{2}-2 w^{H} r+w^{H} R_{x x} w\right. \\
& J(w)=D-2 w^{H} r+w^{H} R_{x x} w
\end{aligned}
$$

$\mathrm{J}(\mathrm{w})$ is called cost function and $D=E\left[|d|^{2}\right]$

We can use the gradient method to locate the minimum value of eq. 15

$$
\nabla_{w}(J(w))=2 R_{x x} w-2 r
$$

The minimum occurs when the gradient is zero, so that the solution for the weights is the optimum Wiener solution as given by

$$
w_{\text {opt }}=R_{x x}^{-1} r
$$

The solution of eq. 17 is contingent upon our knowledge of all signal statistics and thus in the calculation of the array correlation matrix, $R_{x x}$. Since we do not know the signal statistics, we resort to estimating $R_{x x}$ as well as the signal correlation vector over a range of snapshots. The instantaneous estimates of these values are given as

$$
\begin{aligned}
& R_{x x}(t) \approx x(t) x^{H}(t) \\
& r(t) \approx d^{*}(t) x(t)
\end{aligned}
$$

The iterative technique called steepest descent can be used to approximate the gradient of the cost function. The direction of the steepest descent is in the opposite direction as the gradient vector. The method of the steepest descent can be approximated in terms of the weights using the Least Mean Squares method contained in [1]

$w(t+1)=w(t)-\frac{1}{2} \mu \nabla_{w}(J(w(t)))$ 20

where, $\mu$ is the step- size parameter and $\nabla_{w}$ is the gradient of the cost function. The gradient of the cost function is given in eq. 16. If we substitute the instantaneous correlation approximations we obtain the least mean square solution.

$$
\begin{aligned}
& w(t+1)=w(t)-\mu\left[R_{x x} w-r\right]=w(t)+\mu e^{*}(t) x(t) \\
& e(t)=d(t)-w^{H}(t) x(t)=\text { Error signal }
\end{aligned}
$$

The convergence of the LMS algorithm in eq. 21 is directly proportional to the step-size parameter, $\mu$. Too small a step-size will lead to overdamped case because of rather slow convergence. If the convergence is slower than the changing directions of arrival, it is possible that the adaptive array may not acquire the desired signal fast enough to track the ever-changing signal from mobile terminals. On the other hand, if the step-size is too large, the LMS algorithm will over shoot the optimum array weights desired. This is a case of overdamping. If convergence is too fast, then the weights will oscillate about the optimum values so that accurate signal tracking will not be achieved. Stability therefore is assured provided that

$$
0 \leq \mu \leq \frac{1}{2 \lambda_{\max }}
$$

$\lambda$ is the largest eigenvalues of the correlation matrix, $R_{x x}$. Since the correlation matrix is positive, all eigenvalues are positive. Given that all the interfering signals are noise and there is only one desired signal, the condition stated in eq. 22 may be approximated as

$$
0 \leq \mu \leq \frac{1}{2 \operatorname{trace}\left[R_{x x}\right]}
$$




\section{Simulation Results}

The MUSIC technique is simulated using Matlab. Performance of the algorithm has been analyzed by considering mean squared error for a number of trials as a function of number of array elements, distance between elements and snapshots. The simulation has been run for four signals coming from different angles of 14, 28, 35, and 55 degrees for up to 100 snapshots and between 8 and 32 array elements.

To run the Matlab, it has to be ensured that all the files regarding the music algorithm are in the same directory. The main program is run in Matlab. Entered are the number of elements, distance between elements, and number of desired signals with their corresponding angles of arrival in degrees. The program will give the estimated number of signals as output along with the array pattern plots. All that is needed is to obtain the values of the highest $d$ peaks to get the direction of arrival [2]

Test input 1: enter the value for number of signals as 1 . The angle is changed from between 14 to 55 degrees as earlier stated. Sharp peaks will be observed for each signal.

Test input 2: enter the value for the number of signals as 4. Enter values of four angles representing each of the four signals. The outputs are observed.

There are a number of parameters for which changes can be made and varying results obtained. They are [3]

- $\quad$ Number of elements: 8, 16, 24, 32.

- Distance between elements: $0.1 \lambda, 0.2 \lambda, 0.3 \lambda, 0.5 \lambda$

- Number of snapshots/samples: 100 to 1000

- Number of signals: 1 to 4

- $\quad$ Angles of arrival: $14^{\circ}, 28^{\circ}, 35^{\circ}, 55^{\circ}$

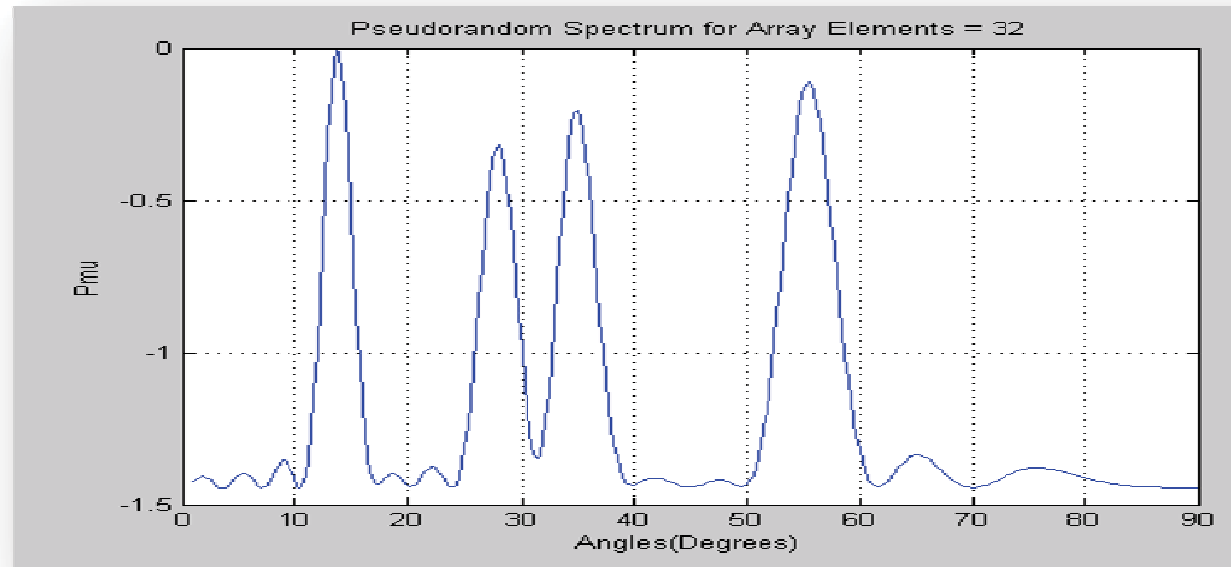

Fig. 6.10: Plot for estimated directions of arrival $\theta=14^{\circ}, 28^{\circ}, 35^{\circ}, 55^{\circ}$ along the peak of music spectra for 32-element array

In the case of Beam Pattern Synthesis, the system continuously updates the data sampled from the sensors of the array. The processing of this data is conducted at periodic intervals in order to estimate the direction of arrival and related information regarding the signals and interferers via the music algorithm. The estimated information is then applied in the calculation of the array 
element complex weights. The snapshots stored in the memory storage and the computed complex weights updates are synchronized. Finally, the stored data is weighted with the updated complex weights in a beamformer to give desired array pattern.

The parameters for which changes can be made are just two, namely the angle of the desired signal and that of the interferer. Simulations shall be made for one desired signal and one interfering signals as follows: Desired signal direction of arrival $=0^{\circ}$ Interfering signal direction of arrival $=60^{\circ}$ Number of array elements $=16$ Spacing between array elements $=0.2 \lambda$

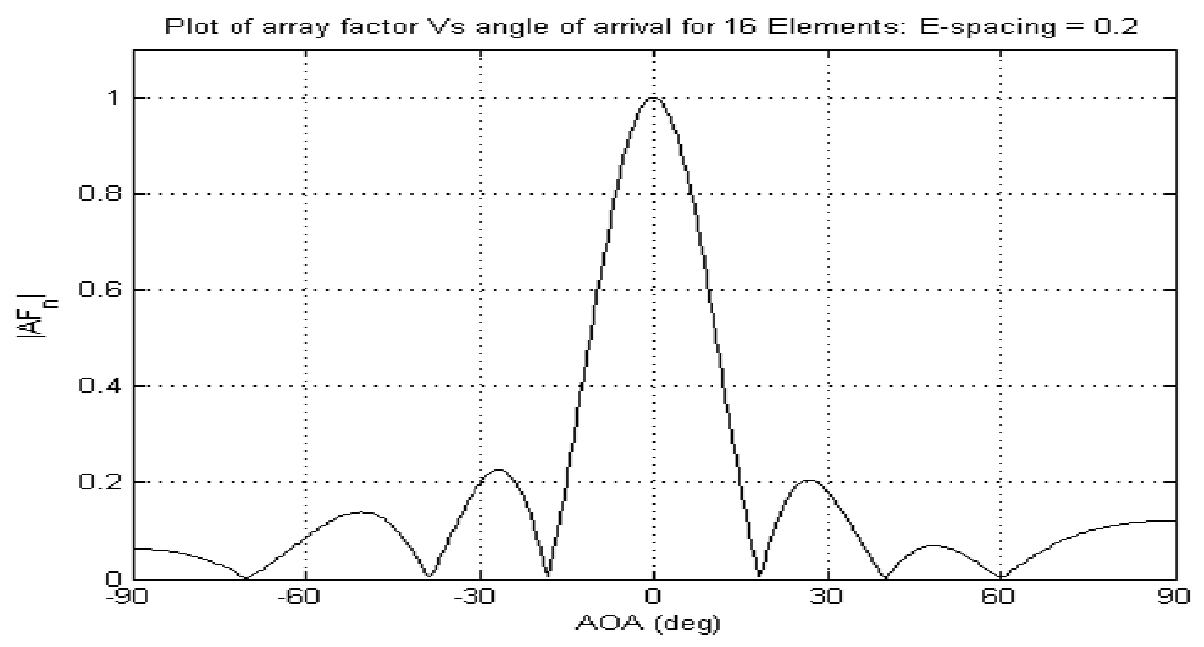

Fig. 6.55: Plot of weighted Least Mean Square Array versus Angle of Arrival

\section{Conclusion}

Null steering and pattern control as well as direction of arrival estimation were carried out in this presentation. The music algorithm for direction of arrival estimation and least mean squares algorithm for null steering and pattern control have been implemented. The fundamental problem of interference has therefore been dealt with. From this paper, it can be made certain that the techniques adopted for direction of arrival estimation and null steering can be applied to interference suppression in an interference prone high performance mobile communication. Now, having overcome this problem of interference by nulling, it is possible to improve upon the capacity of a gsm system.

\section{References}

Frank Gross "Smart Antennas for Wireless Communications". McGraw-Hill 2005

Nikhil Shetty "Direction of Arrival Estimation Algorithms" 2007

Nsionu Ifeanyi "Improving gsm Network Capacity using Adaptive Smart Antenna System 